\title{
Fonf Theodore Harold Maiman
}

Depuis plus de 50 ans, la communauté scientifique peine à trancher cette question : qui a inventé le laser ? Le premier brevet a été déposé par G. Gould, on a retrouvé la première mention de l'idée dans la thèse de doctorat du soviétique V.A. Fabrikant en 1940, identifié le premier article scientifique, de C. Townes et A. Schawlow, en 1958, et, plus loin encore, la théorie fondatrice est celle d'Einstein... S'il faut « compter les points », les concepts et choix d'architecture sont donc nés de l'imagination éclairée de plusieurs grands esprits et ils ont chacun contribué à la genèse du laser. Mais, une information est sûre et avérée : Theodore Harold Maiman a construit le premier laser. Pourtant sa principale contribution est probablement ailleurs, dans son génie expérimental et son approche pragmatique d'une problématique complexe, en montrant qu'un laser peut être un système simple.

$\mathrm{T}$ heodore Harold Maiman naît le 11 juillet 1927 à Los Angeles. Son père, Abraham, est un ingénieur en électronique et un inventeur prolifique qui l'initie très jeune au monde de la technologie. $\mathrm{Ce}$ "Géo Trouvetou», qui travaille pour de grandes entreprises de télécoms comme AT\&T ou les Bell Telephone Laboratories, entretient soigneusement un petit laboratoire d'électronique dans sa cave, où le jeune Theodore découvre la science expérimentale. C'est un enfant turbulent, probablement hyperactif, à l'intelligence moyenne. Ses professeurs l'identifient même comme un fauteur de troubles, mais lui reconnaissent un naturel fonceur et persévérant. Peu après la naissance de son fils, Abraham obtient un poste à Denver, dans le Colorado, et la famille s'installe chez les grands-parents maternels.

Maiman décroche son premier job à 12 ans, dans un atelier de réparations électroniques où il se rend après les cours. D'abord sceptique sur l'aptitude du jeune garçon à dépanner des appareils, le propriétaire le charge du ménage de l'atelier. Mais peu de temps après, devant l'aisance manifeste de Theodore face aux problèmes d'électronique, il se décide à lui confier quelques dossiers. Cet atelier devient très vite la seconde maison de Maiman : il y travaille plusieurs soirs par semaine, plusieurs années de suite.

\section{Les études}

À 17 ans, Maiman propose sa candidature à la US Navy. Il est admis dans le programme de formation aux radars et communications, qui lui permet notamment de parfaire ses connaissances en électro- nique. Après un court passage à la National Union Radio où il teste des tubes à vide, il s'inscrit à l'université du Colorado et décroche un Bachelor en physique de l'ingénieur. II poursuit ses études à la Columbia University de New York, dans la filière Electrical Engineering, et parvient ensuite à intégrer le département de physique de la prestigieuse Stanford University de Californie. Il y fait sa thèse de doctorat sous la direction de Willis E. Lamb [1913-2008], brillant théoricien et futur Prix Nobel. Le projet vise la démonstration du décalage de Lamb, le très faible déplacement d'un niveau atomique élevé de l'hélium. La mise au point de l'expérience nécessite un soin minutieux, les manipulations et les acquisitions sont extrêmement pointues, mais Maiman est à la hauteur du défi et montre un formidable talent de technicien et d'expérimentateur. Il obtient son doctorat de physique en 1955.

\section{Premiers travaux scientifiques}

Peu intéressé par une carrière académique, Maiman s'octroie en août 1955 un long voyage pour prendre le temps de réfléchir et, à son retour, il rejoint Lockheed Aerospace qui recrute largement en prévision d'un gros contrat du gouvernement. Mais, contre toute attente, le contrat est décroché par un concurrent, Martin Marietta, et Maiman se retrouve désœuvré. Grâce à son ami Irwin L. Hahn (né en 1921), professeur à Berkeley, il entre aux Hughes Research Laboratories à Culvert City, en Californie. Ce centre de recherche est un creuset d'innovation scientifique et technologique particulièrement fécond ; Mai-

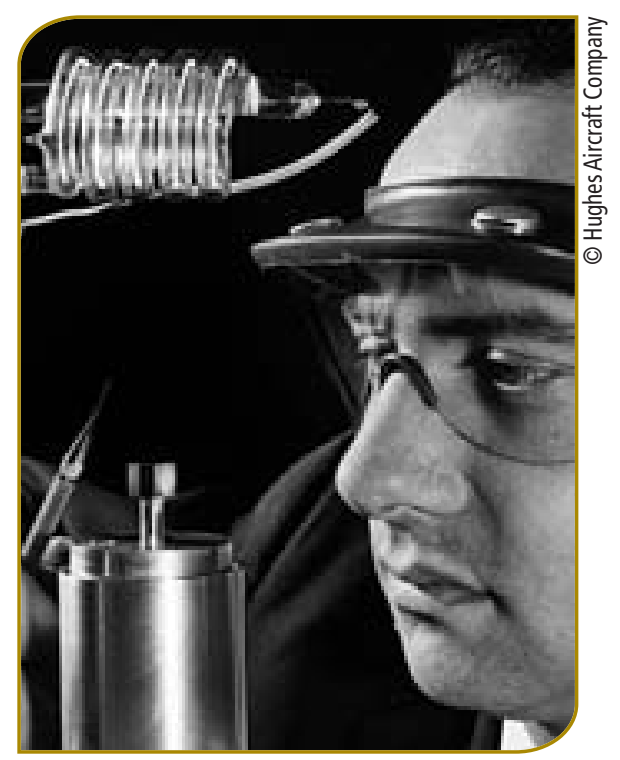

man est affecté au département de physique atomique. À cette époque, le développement des masers inventés par Charles Townes (né en 1915) fait l'objet d'une rude compétition, et on confie à Maiman la mise au point d'un maser compact et embarquable. Comme dans ses travaux avec Lamb, il relève le défi et transforme les gigantesques masers - des mastodontes de laboratoire qui pèsent plus de deux tonnes - en de petits bijoux à base de rubis artificiel.

\section{Le laser !}

Après ce succès, il s'intéresse au laser dont le concept, basé sur les travaux théoriques d'Einstein et formalisé par Charles Townes et Arthur Schawlow [19211999], est vieux d'à peine un an. Aucune application n'est encore envisagée, mais le projet est largement subventionné aux Etats-Unis, notamment par le département de la Défense. 
Depuis peu, les recherches, menées dans de prestigieux centres (Bell Labs, IBM...), se sont cristallisées autour du choix d'un matériau capable de stocker l'énergie lumineuse et de la rendre sous stimulation. Mais, suite à une erreur de mesures, le rubis a été classé «hors compétition » et c'est probablement là le coup de chance de Maiman... car il connaît ce matériau : il l'utilise pour son maser miniature et ses propres tests lui indiquent qu'un laser à rubis est viable si on l'éclaire avec une lampe suffisamment puissante.

Son assistant, Charles Asawa, a l'idée d'utiliser l'éclair bref et intense d'un flash de photographe et l'équipe tente ainsi d'emblée l'approche si efficace d'une excitation pulsée...

Maiman dessine et conçoit sa structure, comme toujours simple et pragmatique : un barreau de rubis (recyclé de ses travaux sur le maser) entouré d'un tube flash et inséré dans un cylindre en aluminium. Et, pour former la cavité optique, Maiman choisit de déposer deux couches d'argent sur les faces du barreau de rubis, en prenant soin de percer un petit trou dans l'un de ces miroirs pour ménager un passage au futur faisceau de lumière cohérente. Ainsi, alors que les Hughes Labs ne participent pas vraiment à la course au laser qui émeut le pays et ne soutiennent donc que chichement ce projet, Maiman et son équipe, en véritables outsiders, vont créer la surprise car personne, absolument personne, ne s'attend à les voir concourir - et gagner!

Le 16 Mai 1960, après avoir vérifié et revérifié que tout est en ordre, Maiman branche le courant et, en augmentant la puissance du flash excitateur, observe l'émission du barreau de rubis atteindre progressivement le seuil d'oscillation. Après neuf mois d'effort, neuf mois seulement, il obtient ainsi (et du premier coup !) la première émission laser.

\section{Contrecoups et amertume}

Mais, on ne crée pas impunément la surprise et, immédiatement après ce tour de force, les problèmes commencent.

Maiman soumet ses résultats aux prestigieuses Physical Review Letters où le rapporteur rejette l'article en commentant sa décision d'un laconique «encore un papier sur le maser». Peut-être a-t-il jugé l'auteur trop extérieur au cercle des initiés...

Maiman tente alors sa chance auprès de la non moins fameuse revue Britannique Nature, qui le publie le 6 août 1960 en donnant à ce papier historique un titre pour le moins modeste : on ne parle pas de laser, mais d' ' un rayonnement optique stimulé dans le rubis".

Toutefois, entre la découverte et la publication, le risque existe de se faire voler la vedette et Howard Hughes, en fin politique et grand homme d'affaires, convoque une conférence de presse le 7 juillet à New York et revendique pour Maiman la paternité du premier laser. C'est un succès retentissant. Très vite, le laser à rubis est dupliqué et d'autres sont inventés : à gaz, à électrons libres, pour le visible, I'infrarouge, continu ou pulsé... Les fonds ne manquent pas et les scientifiques font preuve d'une imagination sans limites. Maiman, quant à lui, croit en l'avenir industriel du laser et quitte les Hughes Labs pour fonder successivement la Korad Corporation (1961), la société de conseil Maiman Associates (1968) et la Laser Video Corporation (1972). En 1976, il est nommé vice-président chargé du développement technologique de la firme TRW (devenue depuis lors la Northrop Grumman Corporation).

Mais Maiman se sent hors du cadre. L'intelligentsia scientifique le perçoit comme un outsider chanceux, trop original, pas légitime et lui réserve pour longtemps une place à l'écart.

D'ailleurs, le Prix Nobel de 1964 qui récompense la genèse du laser "oublie» Maiman : au sens strict, il n'a pas inventé le laser. Malgré trois nominations, il ne I'obtient jamais et un sentiment d'injustice - partagé par certains - s'empare de Maiman. Ce déni de paternité du Comité Nobel, il le perçoit comme un manque de reconnaissance de toute la communauté et l'amertume du génial technicien transparaît dans son livre autobiographique, The Laser Odyssey, publié en 2000.

\section{La gloire}

Pourtant, Maiman reste celui qui a construit le premier laser et de nombreuses ins-

\section{Principales dates}

11 juillet 1927

Naissance à Los Angeles (Californie, Etats-Unis)

1955 Doctorat de physique à Stanford

1959 Mise au point d'un maser compact à rubis

16 Mai 1960 Invention du laser aux Hughes Labs $1983 \quad$ Wolf Prize of Physics

1984 Intronisation au National Inventors Hall of Fame

1987 Japan Prize

5 mai 2007

Décès à Vancouver (Colombie Britannique, Canada)

titutions du monde entier lui décernent leurs récompenses les plus prestigieuses: récipiendaire de la Ballantine Medal du Franklin Institute en 1962, du Fanny and John Hertz Foundation Award qui lui est remis par le président des Etats-Unis Lyndon B. Johnson en 1966, du Wood Prize de l'Optical Society of America en 1976, du Wolf Physics Prize en 1983, du Japan Prize en 1987 ; Maiman est également membre de plusieurs National Academies of Science and Engineers, fellow des sociétés américaines de physique et d'optique, membre honoraire du prestigieux Royal College of Surgeons of England... Et en 1984, honneur considérable, il est intronisé au National Inventors Hall of Fame. En 1999, il s'installe définitivement à Vancouver, au Canada, en compagnie de sa seconde épouse Kathleen et y mène une activité de consultant indépendant pendant plusieurs années. Sa force créatrice ne faiblira jamais : à l'instar de son père, il convertit une pièce de sa maison en laboratoire de physique et continue d'inventer des objets (parmi lesquels un projecteur TV et un gyroscope) à base de lasers...

Il décède le 5 mai 2007, des suites d'une maladie génétique.

\section{$>$ Riad HAIDAR \\ Onera \\ haidar@onera.fr}

\section{Références :}

[1] The laser odyssey, Th. H. Maiman, Laser Press (2000).

[2] Beam: the race to make the laser, Jeff Hecht, Oxford University Press (2005). 